\title{
Review
}

\section{Smart nanoparticles improve therapy for drug- resistant tumors by overcoming pathophysiological barriers}

\author{
Jian-ping LIU ${ }^{1,2}$, Ting-ting WANG ${ }^{2}$, Dang-ge WANG ${ }^{2}, A n-j i e ~ D O N G{ }^{1, *}$, Ya-ping $\mathrm{LI}^{2}$, Hai-jun $\mathrm{YU}^{2, *}$ \\ ${ }^{1}$ Department of Polymer Science and Technology, Key Laboratory of Systems Bioengineering of the Ministry of Education, School of \\ Chemical Engineering and Technology, Tianjin University, Tianjin 300072, China; ${ }^{2}$ State Key Laboratory of Drug Research \& Center of \\ Pharmaceutics, Shanghai Institute of Materia Medica, Chinese Academy of Sciences, Shanghai 201203, China
}

\begin{abstract}
The therapeutic outcome of chemotherapy is severely limited by intrinsic or acquired drug resistance, the most common causes of chemotherapy failure. In the past few decades, advancements in nanotechnology have provided alternative strategies for combating tumor drug resistance. Drug-loaded nanoparticles (NPs) have several advantages over the free drug forms, including reduced cytotoxicity, prolonged circulation in the blood and increased accumulation in tumors. Currently, however, nanoparticulate drugs have only marginally improved the overall survival rate in clinical trials because of the various pathophysiological barriers that exist in the tumor microenvironment, such as intratumoral distribution, penetration and intracellular trafficking, etc. Smart NPs with stimulusadaptable physico-chemical properties have been extensively developed to improve the therapeutic efficacy of nanomedicine. In this review, we summarize the recent advances of employing smart NPs to treat the drug-resistant tumors by overcoming the pathophysiological barriers in the tumor microenvironment.
\end{abstract}

Keywords: solid tumors; chemotherapy; drug resistance; pathophysiological barriers; tumor microenvironment; nanomedicine; smart nanoparticles

Acta Pharmacologica Sinica (2017) 38: 1-8; doi: 10.1038/aps.2016.84; published online 29 Aug 2016

\section{Introduction}

Chemotherapy is the standard of care for the treatment of advanced or metastatic tumors. However, its success is severely hampered by intrinsic or acquired drug resistance. Insufficient drug delivery to deep tumors and continuous exposure of cancer cells to sublethal doses of cytotoxic drugs are two of the main factors that promote acquired drug resistance ${ }^{[1,2]}$. Solid tumors are highly heterogeneous ${ }^{[3,4]}$, which are surrounded by a dense extracellular matrix (ECM) and lack lymphatic drainage. These factors synergistically induce a reduced transcapillary pressure gradient and an elevated interstitial fluid pressure (IFP), which restricts drug distribution within the tumor ${ }^{[5,6]}$. Moreover, accumulating evidence indicates that the drug-resistant tumor cells produce more acidic vesicles than normal cells. Weakly basic chemothera-

\footnotetext{
* To whom correspondence should be addressed.

E-mail ajdong@tju.edu.cn (An-jie DONG); hjyu@simm.ac.cn (Hai-jun YU)

Received 2016-05-06 Accepted 2016-06-13
}

peutics (eg, doxorubicin, DOX) are therefore entrapped in the acidic vesicles. They are eventually eliminated out of the cells through the secretory pathway, suppressing their antitumor activity $^{[7]}$.

In the past few decades, advancements in nanotechnology have opened up promising opportunities for improving the efficacy and specificity of drug delivery to the tumor lesions. Compared with the free drug form, drug-loaded nanoparticles (NPs) between 10 and $200 \mathrm{~nm}$ in diameter exhibit several advantages, including the following: a prolonged circulating half-life, enhanced tumor distribution, increased cellular accumulation and the capacity to integrate multiple regimens and thereby facilitate combination therapy ${ }^{[8-11]}$. Solid tumors have leaky and permeable blood vessels compared to normal blood vessels. This allows extravasation of the large molecules or NPs owing to the enhanced permeability and retention (EPR) effect of the tumors ${ }^{[12]}$. In addition, the ligand modification of the NPs improves the specificity of their drug delivery to the tumors $^{[13]}$. Despite nanoparticulate drugs displaying obvious advantages over free drugs, the tumor-specific accumulation 
and retention of the drug-loaded NPs is highly dependent on the increased permeability of tumor blood vessels. Before reaching the target site and performing their antitumor function, the drug-loaded NPs need to overcome a complex series of pathophysiological barriers, including limited tumor distribution, restricted intratumoral diffusion, and low efficiency of cellular uptake and intracellular trafficking ${ }^{[14,15]}$. All of these factors synergistically restrict the therapeutic outcomes of nanoparticulate drugs ${ }^{[16,17]}$. In the past few years, smart NPs with stimulus-responsive chemo-physical properties have been extensively developed for cancer nanomedicine. In this review, we have summarized the recent advances in smart nanocarriers for treatment of drug-resistant tumors by overcoming the pathophysiological barriers in both the extracellular and the intracellular microenvironments.

\section{Overcoming extracellular barriers using nanomedicine}

It is well-known that NPs with an average particle size of 100 $\mathrm{nm}$ can be long-lasting in the circulation and then passively accumulate at a leaky site of the tumor vasculature through the "EPR" effect ${ }^{[18]}$. Several recent studies have revealed that NPs between 20 and $60 \mathrm{~nm}$ can permeate deep tumor tissue more efficiently than NPs with larger diameters ${ }^{[19-21]}$. To achieve sufficient drug delivery into the entire tumor, one rational strategy is to design drug-loaded NPs that adapt their sizes within the tumor microenvironment. They must also maintain structural integrity while in the systemic circulation to prevent drug leakage or filtration by the kidneys ${ }^{[22-24]}$. For this purpose, extensive investigations have been performed on smart NPs that respond to biological stimuli in the tumor microenvironment. The stimuli tested so far include deregu-

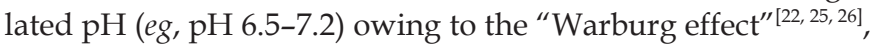
an elevated redox potential ${ }^{[27,28]}$, or overactivated matrix metalloproteinases (MMPs) in multiple stages of cancer progression $^{[2,30]}$.

\section{Overcoming tumor drug resistance with acid-responsive NPs}

To improve the therapeutic efficacy of cancer chemotherapy, extensive efforts have been devoted to developing drug-loaded acid-activatable nanocarriers in the past few decades ${ }^{[31-33]}$. These NPs undergo particle size change ${ }^{[34]}$, surface charge status switching ${ }^{[35]}$, or a hydrophobic to hydrophilic transition ${ }^{[36]}$ by responding to the $\mathrm{pH}$ drop in the weakly acidic microenvironment of tumor or cancer cells. For instance, Wang et al recently reported a novel set of intelligent cluster (iCluster) NPs to improve the tumor penetration and distribution of the poly(amidoamine) (PAMAM) prodrug ${ }^{[3]}$. The NPs were composed of two distinct components. The first was a cisplatin prodrug-conjugated PAMAM dendrimer, which was grafted onto a polycaprolactone (PCL) homopolymer via an acid-labile linker 2-propionic-3-methylmaleic anhydride (CDM) to obtain the PCL-CDM-PAMAM/Pt conjugate. The second component of the NPs was a poly(ethylene glycol)-b-poly(e-caprolactone) (PEG-b-PCL) diblock copolymer (Figure 1A), which controlled the particle size and stabilized the NPs by providing a PEG corona and a hydrophobic PCL core. The PAMAM prodrugconjugated NPs maintained good integrity and demonstrated a hydrodynamic particle size of approximately $100 \mathrm{~nm}$ during blood circulation. The NPs were designed to enhance the tumor accumulation of the PAMAM prodrug through the EPR effect, while releasing the PAMAM prodrug in response to the acidic tumor environment (ie, $\mathrm{pH}$ 6.5-7.2). The PAMAM prodrug penetrated deeply into the tumor, owing to the small particle size $(\sim 5 \mathrm{~nm})$. Subsequently, the prodrug performed its antitumor function by being converted to cisplatin in the reducing environment of the cytosol (Figure 1B) ${ }^{[37]}$.

In a multilayer spheroidal cancer (MSC) model derived from BxPC-3 human pancreatic cancer cells, the hydrophobic PCL core (with red fluorescence labeling) of the iCluster NPs was observed to attach to the periphery of MCSs without a noticeable distribution in the internal area, after a $24 \mathrm{~h}$ incubation at

A

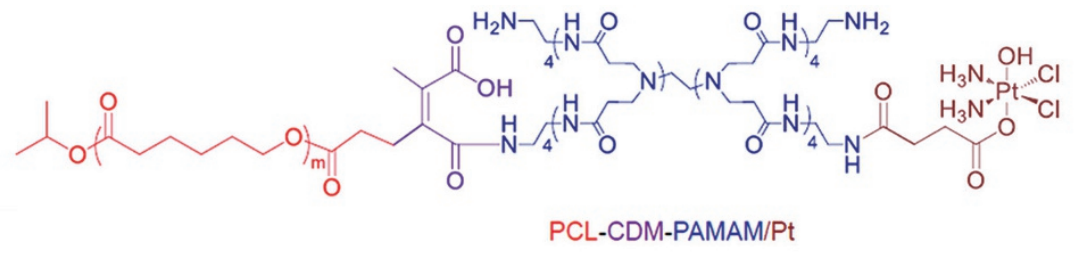

B
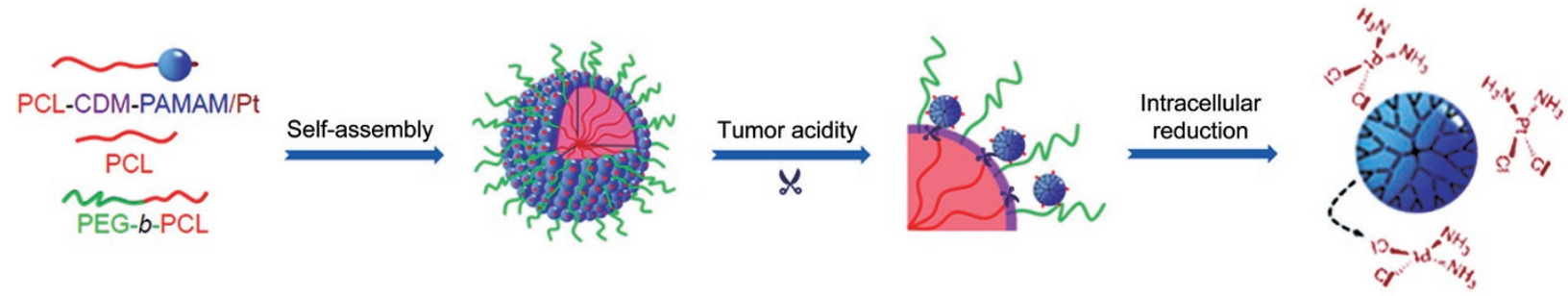

Figure 1. (A) Chemical structure of the acid-responsive PCL-CDM-PAMAM/Pt conjugate. (B) Self-assembly of acid/redox dual-responsive iCluster/Pt NPs. Adapted with permission from [37]. Copyright (2016) National Academy of Sciences. 
a $\mathrm{pH}$ of 6.8. In contrast, the green fluorescence labeling of the PAMAM prodrug was found in the core of the tumor sphere. This indicated that the PAMAM prodrug was released from the iCluster NPs in acidic conditions, which mimic the acidic tumor microenvironment, and penetrated deeply into the tumor. The antitumor efficacy of the prodrug-conjugated NPs was evaluated in a tumor xenograft model of cisplatinresistant A549 (A549R) lung cancer in vivo. The iCluster NPs displayed much higher antitumor efficacy than the nonresponsive control, which can be attributed to the ability of the NPs to release the PAMAM prodrug in the acidic environment, where it had good tumor penetration ${ }^{[20,21,38]}$.

\section{Overcoming the extracellular barrier with protease-activatable NPs}

Hydrophilic molecular modification such as PEG shielding has been thoroughly employed to improve the stability and pharmacokinetics of drug-loaded NPs ${ }^{[39]}$. PEG shielding reduces the nonspecific interaction of NPs with blood components and non-targeted tissues, prolongs circulation time and decreases toxic effects. However, stable PEG modification appears to suppress the intratumoral diffusion and cellular uptake of the carriers. This remains another notable extracellular barrier for cancer nanomedicine ${ }^{[30]}$. It is apparent that PEG shielding must be more dynamic to improve the efficacy of nanoparticulate drugs. The NPs need to be stabilized with the PEG corona for prolonged circulation in the blood. However, after reaching the tumor site, the PEG corona should be removed for efficient tumor penetration and intracellular trafficking. This can be achieved by attaching a PEG shell to the NPs via stimuluslabile linkages.

MMPs, in particular, MMP-2/9 are over-activated in multiple stages of human cancers. They serve as key effectors involved in tumor angiogenesis, invasion, and metastasis ${ }^{[40,41]}$. MMP-responsive nanocarriers or imaging reagents have been developed to improve tumor accumulation and retention ${ }^{[30,42]}$. Torchilin et al recently reported a prodrug-loaded smart nanocarrier for cancer therapy ${ }^{[43]}$. The drug-loaded NPs were selfassembled from an octapeptide (GPLGIAGQ)-linked PEG2000 paclitaxel (PTX) prodrug and a TAT-functionalized PEG1000phosphoethanolamine (PE) amphiphilic lipid. The octapeptide is an MMP-2-cleavable spacer that is able to release PTX in the tumor microenvironment. TAT is an arginine-rich oligopeptide with a superior tissue penetration capability ${ }^{[4]}$. The resulting NPs were covered by a hydrophilic PEG shell with PTX located in the hydrophobic core. The TAT peptide was shielded with a PEG2000 corona to prevent non-specific interactions with healthy tissues during circulation in the blood. Upon systemic administration, the NPs displayed prolonged circulation in the blood due to the presence of the PEG corona, which passively accumulated in the tumor via the EPR effect. PEG2000 was cleaved in the tumor via MMP-2-induced cleavage of the oligopeptide spacer. Successively, PTX was liberated and the TAT peptide was exposed to facilitate the intratumoral penetration and internalization of PTX. In an A549 human lung cancer xenograft model, the MMP-activatable
NPs resulted in a 2.5-fold higher PTX distribution in the tumor and much higher antitumor efficacy than the non-responsive counterpart. The in vivo tumor distribution and antitumor data consistently exhibited the clinical potential of the MMPactivatable NPs for the treatment of the drug-resistant tumors by overcoming the extracellular barriers.

\section{Combating the drug-resistant tumor by priming the tumor microenvironment}

The strategies described above are focused on the treatment of the drug-resistant tumor by designing tumor microenvironment-adaptable NPs. However, the activation of these smart NPs is passively dependent on pre-existing biological signals. Because tumors are highly heterogeneous, the clinical application of drug-loaded NPs is limited. Therefore, the second section of this review is focused on an emerging paradigm of nanomedicine that actively primes the host microenvironment of solid tumor and cancer cells.

\section{Combating the drug-resistant tumors by degradation of ECM}

The major component of ECM is hyaluronic acid (HA). HA is a linear glycosaminoglycan (GAG), composed of repeating $\mathrm{N}$-acetyl glucosamine and glucuronic acid units. HA accumulation contributes to the increase of IFP in tumors, limiting the intratumoral diffusion of therapeutics. The interaction between HA and cancer cells is closely related to cancer metastasis $^{[45]}$. In addition to stromal HA, many cancer cells have a pericellular HA matrix, which prevents the access of anticancer drugs or drug-loaded NPs. One promising strategy to enhance drug delivery to solid tumors is to degrade HA with biological or physical approaches. For instance, PEGylated recombinant human hyaluronidase ( $\mathrm{rHuPH} 20, \mathrm{PEGPH} 20)$ is undergoing clinical trials, where it is administered $1 \mathrm{~d}$ before applying anticancer agents to ensure the depletion of tumor $\mathrm{HA}^{[46]}$. However, HA that has been the systemically injected is subject to enzyme degradation while circulating and tends to induce muscle spasms and thromboembolism, as reported in clinical studies ${ }^{[47]}$.

Very recently, Cheng et al demonstrated that the HA modification of drug-loaded NPs improved the efficacy of drug delivery by degrading the HA of the tumoral stroma ${ }^{[48]}$. In this study, rHuPH20 was employed to modify the surface of drugloaded poly(lactic-co-glycolic acid)-b-poly(ethylene glycol) NPs (PLGA-PEG-NPs). For this purpose, the drug-loaded (ie, doxorubicin, DOX) bare NPs were sequentially modified with a thiolated rHuPH20 and an extra PEG shell (Figure 2). rHuPH20 conjugated on the surface of NPs showed no influence on the circulation time of the NPs. Meanwhile, the PEG corona maintained the enzyme activity of rHuPH20 in the blood. Compared to rHuPH20 in its free form, rHuPH20 conjugated on the surface of NPs showed a much higher efficacy of NP diffusion into the matrix gel. Additionally, surface rHuPH20 enhanced NP accumulation in 4T1 breast tumor xenografts owing to the increased tumor penetration. The rHuPH20-functionalized PLGA-PEG-NPs exhibited significantly improved antitumor efficacy over their unmodified 

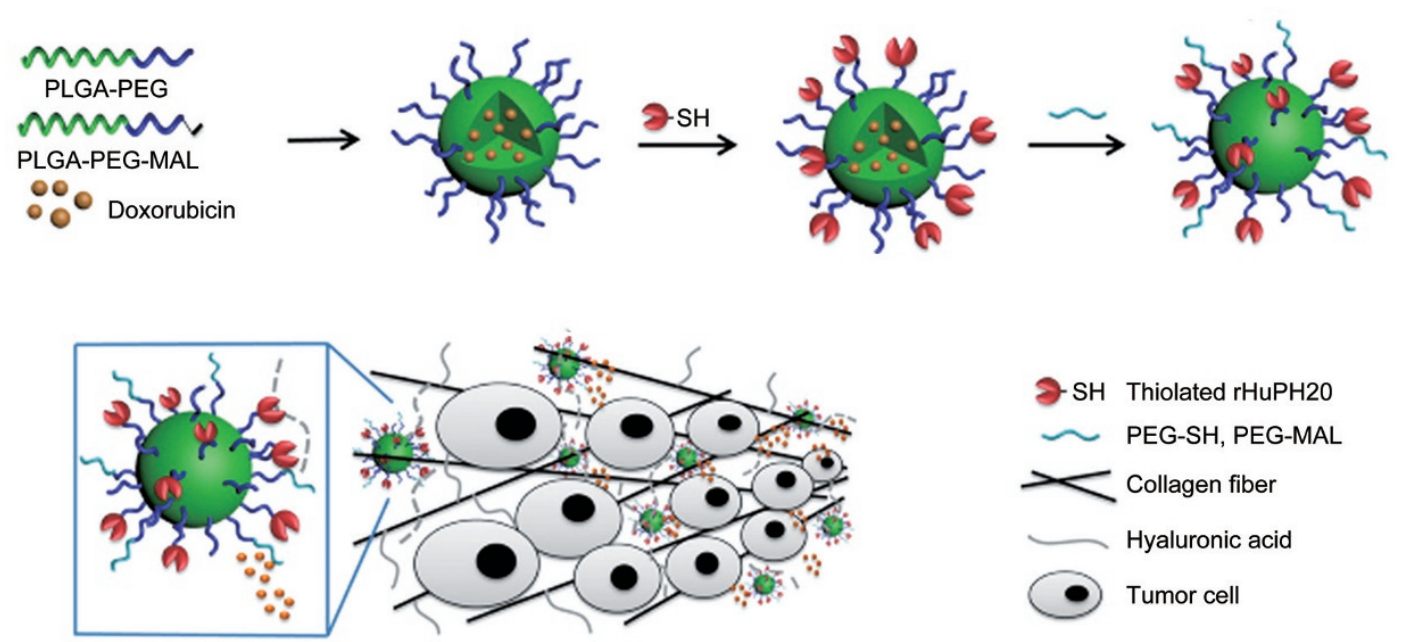

Figure 2. Self-assembly of rHuPH2O-modifed NPs and schematic illustration of enhanced tumor penetration via degradation of HA. Adapted with permission from [48]. Copyright (2016) American Chemical Society.

counterparts. TUNEL staining of the tumor section revealed notable apoptosis of the tumor cells, indicating that the antitumor efficacy of the drug-loaded NPs could be attributed to the cytotoxicity of DOX. This study suggested that the hyaluronidase modification of the drug-loaded NPs was promising for improving the efficacy of cancer nanomedicine. This approach might also enable the clinical translation of NPs, which was previously considered impractical owing to the inefficient diffusion into solid tumors.

Overcoming drug resistance by phototherapy-induced alteration of the tumor microenvironment

As previously mentioned, the inefficient tumor penetration of drug-loaded NPs is the dominant extracellular barrier for nanomedicine. In the past few years, phototherapy, in particular photothermal therapy (PTT), has gained increased attention for combating drug-resistant tumors ${ }^{[49]}$. PTT induces ECM degradation and improves NP delivery to solid tumors by converting near-infrared (NIR) light to local heat. Compared to the conventional modalities for cancer therapy, including surgical resection, chemotherapy and radiotherapy, PTT is noninvasive and induces minimal side effects ${ }^{[50,51]}$. However, the majority of the NPs employed for PTT are inorganic nanomaterials (eg, gold nanorods and carbon nanomaterials). Their non-biodegradability and therefore accumulation in the body remains one of the long term concerns for clinical translation $^{[52]}$. To address the disadvantages of inorganic NPs for PTT of the tumor, organic nanoplatforms with high photothermal conversion efficiency have been studied for PTT and chemotherapy of drug resistant tumors.

Our laboratory reported polymer-based acid and NIR light dual-responsive micelle NPs for the treatment of DOXresistant breast cancer ${ }^{[5]}$. The micelles were composed of a $\mathrm{pH}-$ responsive diblock polymer and a polymeric prodrug of DOX (Figure 3A and 3B). The diblock copolymer was functionalized with Cypate, an NIR dye, for photothermal conversion in the NIR region ${ }^{[53]}$. At physiological $\mathrm{pH}(i e, 7.4)$, the micelles formed a compact structure with a particle size of approximately $30 \mathrm{~nm}$, allowing prolonged circulation in the blood and passive tumor targeting. The micelles quickly dissociated in a weakly acidic environment $(i e, \mathrm{pH} \leq 6.2)$ to release the DOX prodrug (Figure 3C). Upon NIR laser irradiation, the micelles induced a significant hyperthermia effect, which degraded the ECM and triggered tumor penetration of the DOX prodrug (Figure 3D). Consequently, the NIR irradiation-induced hyperthermia effect and the DOX prodrug-conducted chemotherapy coordinated to inhibit the growth of a DOX-resistant MCF-7/ADR breast tumor in an orthotopic tumor model. Our study might suggest that physical stimulus-triggered modulation of the host microenvironment, in this case the hyperthermia effect, can notably improve therapeutic efficacy against drug-resistant tumors by destroying the ECM.

\section{Combating cancer drug resistance by overcoming intra- cellular barriers}

Following accumulation within the tumor and extracellular diffusion, drug-loaded NPs have to bypass several intracellular barriers to exert their antitumor function. The NPs must be internalized into the endocytic vesicles, travel from the vesicles to the cytosol and unload the drug payload (this step can happen in the vesicles or in the cytosol after the NPs escape from the vesicles) ${ }^{[15]}$. Of all of these barriers, cellular uptake and intracellular trafficking are the two most crucial factors affecting the therapeutic outcomes of NP-mediated chemotherapy ${ }^{[54,55]}$. Surface modification with a targeting ligand or cell penetration peptide (CPP) is a practical approach to prompt the internalization of the drug-loaded NPs. For instance, Wang et al demonstrated that CRGDK-modification notably increased the cellular accumulation of liposomal NPs by targeting the Neuropilin-1 (Nrp-1) receptor on the membrane of tumor cells ${ }^{[56,57]}$. An excellent example of a CPP, TAT is an arginine-rich peptide derived from the HIV-1 transcrip- 
A

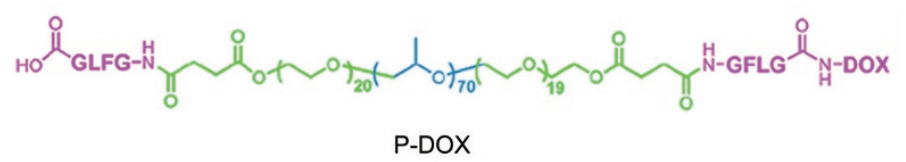<smiles>CC(C)CC(NC(=O)C(Cc1ccccc1)NC(=O)CN)C(=O)NCC(=O)O</smiles>

$\mathrm{H}_{2} \mathrm{~N}-\mathrm{GFLG}-\mathrm{COOH}$

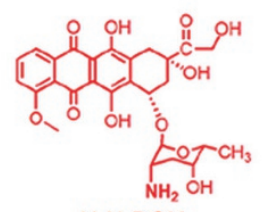

$\mathrm{H}_{2} \mathrm{~N}-\mathrm{DOX}$
B

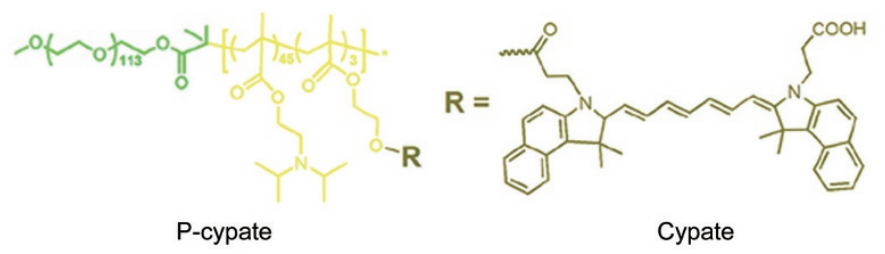

C

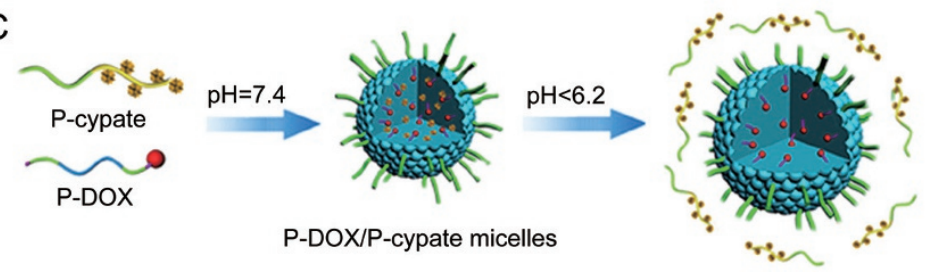

D

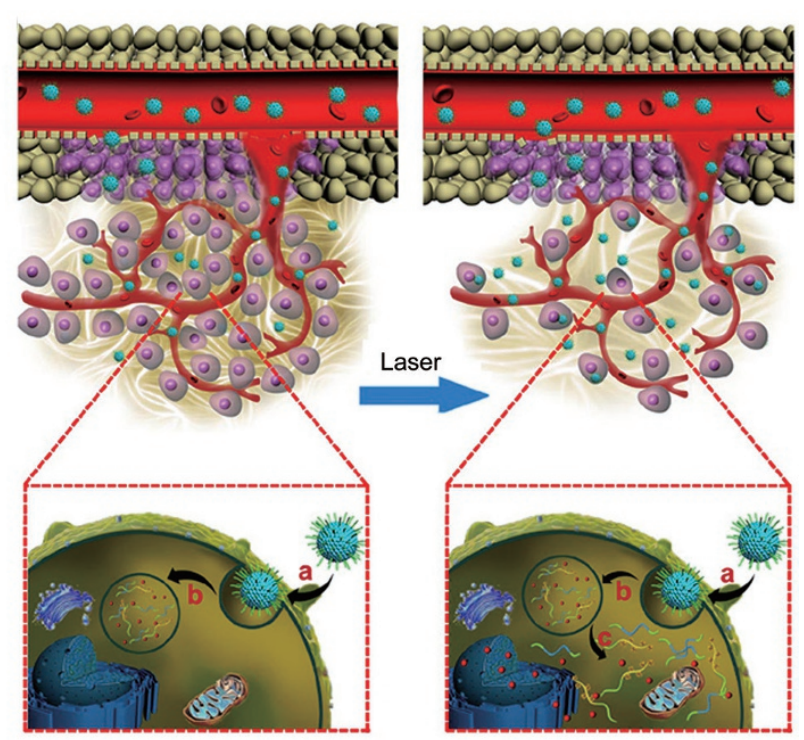

a: Cellular uptake

b: Lysosome dissociation

c: Cytosol release

Figure 3. Chemical structures of (A) DOX prodrug (P-DOX) and (B) Cypate-conjugated P-cypate diblock copolymer. (C) Self-assembly of pH and NIR light dual-responsive P-DOX/P-cypate micelles. (D) Schematic illumination for combating DOX-resistant breast cancer with the pH and NIR light dualresponsive P-DOX/P-cypate micelles (a: Cellular uptake; b: Lysosome dissociation; c: Cytosol release). Adapted with permission from [5]. Copyright (2015) John Wiley \& Sons, Inc.

tion peptide. Attaching TAT has long been identified as a method for improving intracellular uptake of NPs ${ }^{[58,59]}$. The possible mechanism underlying this effect of TAT is an interaction with the negatively charged plasma membrane, which enhances the permeability of the cell membrane, triggering

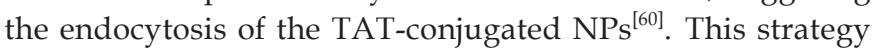
had been employed by Huang et al to combat DOX resistance in breast tumors ${ }^{[6]]}$. A positive surface charge is another critical factor affecting the interaction of NPs with tumor cells. Liposomal NPs bearing a positive surface charge have shown increased cellular uptake compared with their neutral counterparts $^{[62,63]}$.

Following internalization, the intracellular transportation of drug-loaded NPs from the endocytic vesicles to the cytosol or nucleus is another intracellular barrier. In a recent study by Chen et al, an NIR light-responsive NP was developed for the treatment of cisplatin-resistant lung cancer by enhancing the intracellular trafficking of the anticancer drug ${ }^{[64]}$. The NP was prepared by co-assembly of a Cypate-conjugated diblock copolymer with an alkylated cisplatin prodrug (Figure 4). The NPs significantly enhanced tumor accumulation, as well as the cellular uptake of the drug, in cisplatin-resistant A549R cells. NIR laser illumination induced a notable hyperthermia effect at a $\mathrm{pH}$ of 5.0 to promote lysosome disruption, increasing the intracellular trafficking of the $\mathrm{Pt}(\mathrm{IV})$ prodrug into the cytoplasm $^{[65]}$. Meanwhile, PTT inhibited the efflux of cisplatin by suppressing MRP1 expression in the A549R cells. This study indicated the promise of NP-mediated PTT for treating drugresistant tumors by promoting intracellular translocation of the anticancer drugs.

It would be impossible to discuss the enhanced intracellular trafficking of drug-loaded NPs without mentioning photodynamic therapy (PDT). PDT can trigger drug release from NPs or endocytic vesicles by inducing reactive oxygen species (ROS) generation and destabilizing the cytoplasmic membrane ${ }^{[66-69]}$. For example, Lin et al employed nanoscale coordination polymers (NCPs) to construct a PS-loaded hybrid NP. The NCP core was constructed through the coordination of cisplatin prodrugs with zinc metal ions and then coated with an asymmetric lipid bilayer containing a porphyrin-conjugated phospholipid (pyrolipid) ${ }^{[70]}$. The resulting NCP@pyrolipid maintains structural integrity extracellularly but releases cisplatin upon the PDT-triggered vesicle burst and subsequent cytosol reduction of the prodrug. With a combination of cisplatin-mediated chemotherapy and pyrolipid-conducted PDT, the hybrid NPs eradicated a cisplatin-resistant head and neck tumor much more efficiently than each of the therapies singly. 

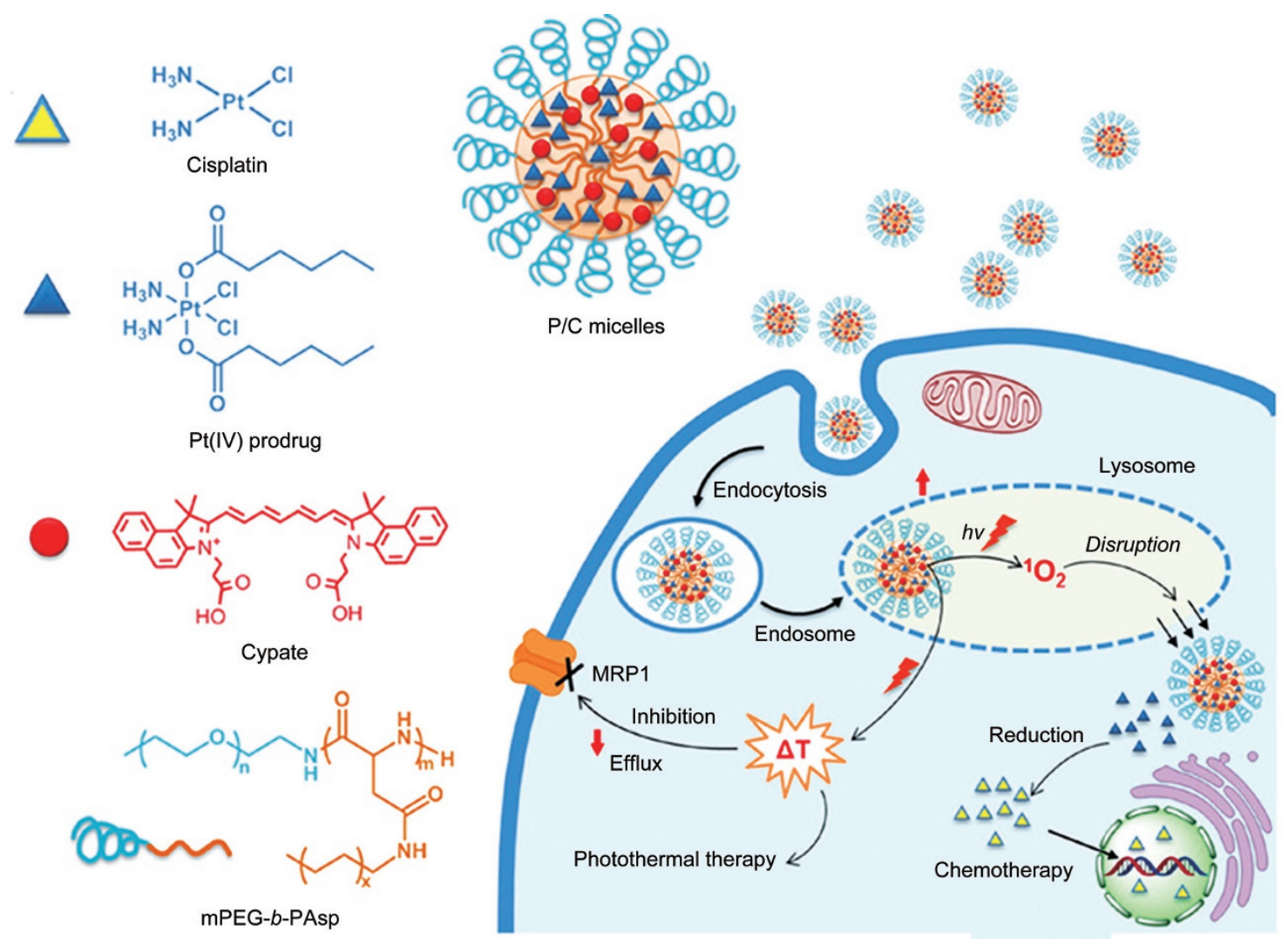

Figure 4. Schematic illustration of the Pt(IV) prodrug and Cypate co-loaded polymeric micelles (P/C-Micelles) for PTT and chemotherapy of cisplatinresistant tumors. Adapted with permission from [64]. Copyright (2016) American Chemical Society.

\section{Conclusion}

Drug-loaded NPs have shown several advantages over the free drug forms, including reduced cytotoxicity, prolonged circulation in the blood and increased accumulation in tumors. Currently, however, nanoparticulate drugs have only marginally improved the overall survival rate in clinical trials because of the various barriers that exist. Intratumoral distribution, penetration and intracellular trafficking are all crucial factors affecting the fate of drug-loaded NPs. In recent years, the advances in nanotechnology have extended cancer nanomedicine beyond conventional knowledge. Smart nanocarriers have been used to improve the therapeutic efficacy of nanoparticulate drugs by overcoming the pathophysiological barriers present in drug-resistant tumors. These novel nanocarriers improve the tumor distribution of the chemotherapeutic by adapting their chemo-physical properties in accordance with the biological stimuli pre-existing at the disease site. Moreover, smart NPs have also been recently proposed for priming the tumor microenvironment for cancer therapy. This set of novel nanocarriers has the potential to improve the therapeutic outcomes of nanomedicine over what has been observed with conventional nanocarriers. In addition to extending our understanding of the biological mechanisms underlying the pathophysiological barriers, a revolution in NP design might translate cancer nanomedicine from a promising strategy to a practical approach for the treatment of drug-resistant tumors in clinical medicine.

\section{Acknowledgements}

The authors sincerely apologize for not being able to cite all the publications related to the current topic owing to space limitations. Financial support from the National Basic Research Program of China (2013CB932704), the National Natural Science Foundation of China (81373359), and the Youth Innovation Promotion Association of CAS (2014248) is gratefully acknowledged.

\section{References}

1 Yin Q, Shen J, Zhang Z, Yu H, Li Y. Reversal of multidrug resistance by stimuli-responsive drug delivery systems for therapy of tumor. Adv Drug Deliv Rev 2013; 65: 1699-715.

2 Damia G, Garattini S. The pharmacological point of view of resistance to therapy in tumors. Cancer Treat Rev 2014; 40: 909-16.

3 Sun XX, Yu Q. Intra-tumor heterogeneity of cancer cells and its implications for cancer treatment. Acta Pharmacol Sin 2015; 36 : 1219-27.

4 Jain RK, Stylianopoulos T. Delivering nanomedicine to solid tumors. Nat Rev Clin Oncol 2010; 7: 653-64.

5 Yu HJ, Cui ZR, Yu PC, Guo CY, Feng B, Jiang TY, et al. pH- and NIR lightresponsive micelles with hyperthermia-triggered tumor penetration and cytoplasm drug release to reverse doxorubicin resistance in 
breast cancer. Adv Funct Mater 2015; 25: 2489-500.

6 Minchinton Al, Tannock IF. Drug penetration in solid tumours. Nat Rev Cancer 2006; 6: 583-92.

7 Webb BA, Chimenti M, Jacobson MP, Barber DL. Dysregulated pH: a perfect storm for cancer progression. Nat Rev Cancer 2011; 11 : 671-7.

8 Davis ME, Chen Z, Shin DM. Nanoparticle therapeutics: an emerging treatment modality for cancer. Nat Rev Drug Discov 2008; 7: 771-82.

9 Peer D, Karp JM, Hong S, FaroKHzad OC, Margalit R, Langer R. Nanocarriers as an emerging platform for cancer therapy. Nat Nanotechnol 2007; 2: 751-60.

10 Torchilin VP. Recent advances with liposomes as pharmaceutical carriers. Nat Rev Drug Discov 2005; 4: 145-60.

11 Liang XJ, Meng H, Wang YZ, He HY, Meng J, Lu J, et al. Metallofullerene nanoparticles circumvent tumor resistance to cisplatin by reactivating endocytosis. Proc Natl Acad Sci U S A 2010; 107: 744954.

12 Maeda H, Nakamura H, Fang J. The EPR effect for macromolecular drug delivery to solid tumors: Improvement of tumor uptake, lowering of systemic toxicity, and distinct tumor imaging in vivo. Adv Drug Deliver Rev 2013; 65: 71-9.

13 Bertrand N, Wu J, Xu XY, Kamaly N, Farokhzad OC. Cancer nanotechnology: The impact of passive and active targeting in the era of modern cancer biology. Adv Drug Deliver Rev 2014; 66: 2-25.

14 Ferrari M. Frontiers in cancer nanomedicine: directing mass transport through biological barriers. Trends Biotechnol 2010; 28: 181-8.

15 Blanco E, Shen H, Ferrari M. Principles of nanoparticle design for overcoming biological barriers to drug delivery. Nat Biotechnol 2015; 33: 941-51.

16 Blum AP, Kammeyer JK, Rush AM, Callmann CE, Hahn ME, Gianneschi NC. Stimuli-responsive nanomaterials for biomedical applications. J Am Chem Soc 2015; 137: 2140-54.

17 Jin ZH, Jin MJ, Jiang CG, Yin XZ, Jin SX, Quan XQ, et al. Evaluation of doxorubicin-loaded $\mathrm{pH}$-sensitive polymeric micelle release from tumor blood vessels and anticancer efficacy using a dorsal skin-fold window chamber model. Acta Pharmacol Sin 2014; 35: 839-45.

18 Matsumura $\mathrm{Y}$, Maeda $\mathrm{H}$. A new concept for macromolecular therapeutics in cancer chemotherapy: mechanism of tumoritropic accumulation of proteins and the antitumor agent smancs. Cancer Res 1986; 46: 6387-92.

19 Jiang W, Kim BYS, Rutka JT, Chan WCW. Nanoparticle-mediated cellular response is size-dependent. Nat Nanotechnol 2008; 3: 14550.

20 Tang L, Fan TM, Borst LB, Cheng JJ. Synthesis and biological response of size-specific, monodisperse drug-silica nanoconjugates. ACS Nano 2012; 6: 3954-66.

21 Cabral H, Matsumoto Y, Mizuno K, Chen Q, Murakami M, Kimura $\mathrm{M}$, et al. Accumulation of sub-100 nm polymeric micelles in poorly permeable tumours depends on size. Nat Nanotechnol 2011; 6: 815-23.

22 Cheng CJ, Bahal R, Babar IA, Pincus Z, Barrera F, Liu C, et al. MicroRNA silencing for cancer therapy targeted to the tumour microenvironment. Nature 2015; 518: 107-10.

23 Wong C, Stylianopoulos T, Cui JA, Martin J, Chauhan VP, Jiang W, et al. Multistage nanoparticle delivery system for deep penetration into tumor tissue. Proc Natl Acad Sci U S A 2011; 108: 2426-31.

24 Guo R, Li LL, Zhao WH, Chen YX, Wang XZ, Fang CJ, et al. The intracellular controlled release from bioresponsive mesoporous silica with folate as both targeting and capping agent. Nanoscale 2012; 4: 3577-83.

25 Webb BA, Chimenti M, Jacobson MP, Barber DL. Dysregulated pH: a perfect storm for cancer progression. Nat Rev Cancer 2011; 11: 671-7.

26 Racker E, Spector M. Warburg effect revisited: merger of biochemistry and molecular biology. Science 1981; 213: 303-7.

$27 \mathrm{Yu} \mathrm{HJ}$, Wagner E. Bioresponsive polymers for nonviral gene delivery. Curr Opin Mol Ther 2009; 11: 165-78.

28 Balendiran GK, Dabur R, Fraser D. The role of glutathione in cancer. Cell Biochem Funct 2004; 22: 343-52.

29 Steeg PS. Tumor metastasis: mechanistic insights and clinical challenges. Nat Med 2006; 12: 895-904.

30 Zhu L, Kate P, Torchilin VP. Matrix metalloprotease 2-responsive multifunctional liposomal nanocarrier for enhanced tumor targeting. ACS Nano 2012; 6: 3491-8.

31 Duan X, Xiao J, Yin Q, Zhang Z, Yu H, Mao S, et al. Smart pH-sensitive and temporal-controlled polymeric micelles for effective combination therapy of doxorubicin and disulfiram. ACS Nano 2013; 7: 5858-69.

32 Ma X, Wang Y, Zhao T, Li Y, Su LC, Wang Z, et al. Ultra-pH-sensitive nanoprobe library with broad $\mathrm{pH}$ tunability and fluorescence emissions. J Am Chem Soc 2014; 136: 11085-92.

$33 \mathrm{Li} \mathrm{J,} \mathrm{Yu} \mathrm{X,} \mathrm{Wang} \mathrm{Y,} \mathrm{Yuan} \mathrm{Y,} \mathrm{Xiao} \mathrm{H,} \mathrm{Cheng} \mathrm{D,} \mathrm{et} \mathrm{al.} \mathrm{A} \mathrm{reduction} \mathrm{and} \mathrm{pH}$ dual-sensitive polymeric vector for long-circulating and tumor-targeted siRNA delivery. Adv Mater 2014; 26: 8217-24.

34 Ju C, Mo R, Xue J, Zhang L, Zhao Z, Xue L, et al. Sequential intraintercellular nanoparticle delivery system for deep tumor penetration. Angew Chem Int Ed Engl 2014; 53: 6253-8.

35 Kim KS, Park W, Hu J, Bae YH, Na K. A cancer-recognizable MRI contrast agents using $\mathrm{pH}$-responsive polymeric micelle. Biomaterials 2014; 35: 337-43.

36 Wang Y, Zhou K, Huang G, Hensley C, Huang X, Ma X, et al. A nanoparticle-based strategy for the imaging of a broad range of tumours by nonlinear amplification of microenvironment signals. Nat Mater 2014; 13: 204-12.

37 Li HJ, Du JZ, Du XJ, Xu CF, Sun CY, Wang HX, et al. Stimuli-responsive clustered nanoparticles for improved tumor penetration and therapeutic efficacy. Proc Natl Acad Sci U S A 2016; 113: 4164-9.

38 Perrault SD, Walkey C, Jennings T, Fischer HC, Chan WCW. Mediating tumor targeting efficiency of nanoparticles through design. Nano Lett 2009; 9: 1909-15.

39 Mickler FM, Vachutinsky Y, Oba M, Miyata K, Nishiyama N, Kataoka $\mathrm{K}$, et al. Effect of integrin targeting and PEG shielding on polyplex micelle internalization studied by live-cell imaging. J Control Release 2011; 156: 364-73.

40 Roy R, Zhang B, Moses MA. Making the cut: Protease-mediated regulation of angiogenesis. Exp Cell Res 2006; 312: 608-22.

41 Roy R, Yang J, Moses MA. Matrix metalloproteinases as novel biomarkers and potential therapeutic targets in human cancer. J Clin Oncol 2009; 27: 5287-97.

42 Olson ES, Jiang T, Aguilera TA, Nguyen QT, Ellies LG, Scadeng M, et al. Activatable cell penetrating peptides linked to nanoparticles as dual probes for in vivo fluorescence and MR imaging of proteases. Proc Natl Acad Sci U S A 2010; 107: 4311-6.

43 Zhu L, Wang T, Perche F, Taigind A, Torchilin VP. Enhanced anticancer activity of nanopreparation containing an MMP2-sensitive PEG-drug conjugate and cell-penetrating moiety. Proc Natl Acad Sci U S A 2013; 110: 17047-52.

44 Torchilin VP, Rammohan R, Weissig V, Levchenko TS. TAT peptide on the surface of liposomes affords their efficient intracellular delivery even at low temperature and in the presence of metabolic inhibitors. Proc Natl Acad Sci U S A 2001; 98: 8786-91.

45 Provenzano PP, Cuevas C, Chang AE, Goel VK, Von Hoff DD, Hingorani SR. Enzymatic targeting of the stroma ablates physical barriers to 
treatment of pancreatic ductal adenocarcinoma. Cancer Cell 2012; 21: 418-29.

46 Simpson MA, Wilson CM, Furcht LT, Spicer AP, Oegema TR Jr, McCarthy JB. Manipulation of hyaluronan synthase expression in prostate adenocarcinoma cells alters pericellular matrix retention and adhesion to bone marrow endothelial cells. J Biol Chem 2002; 277 : 10050-7.

47 Hingorani SR, Harris WP, Beck JT, Berdov BA, Wagner SA, Pshevlotsky $\mathrm{EM}$, et al. Phase lb study of PEGylated recombinant human hyaluronidase and gemcitabine in patients with advanced pancreatic cancer. Clin Cancer Res 2016; 22: 2848-54.

48 Zhou H, Fan Z, Deng J, Lemons PK, Arhontoulis DC, Bowne WB, et al. Hyaluronidase embedded in nanocarrier PEG shell for enhanced tumor penetration and highly efficient antitumor efficacy. Nano Lett 2016; 16: 3268-77.

49 Gormley AJ, Larson N, Sadekar S, Robinson R, Ray A, Ghandehari $\mathrm{H}$. Guided delivery of polymer therapeutics using plasmonic photothermal therapy. Nano Today 2012; 7: 158-67.

50 Idris NM, Jayakumar MKG, Bansal A, Zhang Y. Upconversion nanoparticles as versatile light nanotransducers for photoactivation applications. Chem Soc Rev 2015; 44: 1449-78.

51 Guo C, Yu H, Feng B, Gao W, Yan M, Zhang Z, et al. Highly efficient ablation of metastatic breast cancer using ammonium-tungstenbronze nanocube as a novel $1064 \mathrm{~nm}$-laser-driven photothermal agent. Biomaterials 2015; 52: 407-16.

52 Li Y, Lin TY, Luo Y, Liu Q, Xiao W, Guo W, et al. A smart and versatile theranostic nanomedicine platform based on nanoporphyrin. Nat Commun 2014; 5: 4712.

53 Ye Y, Li WP, Anderson CJ, Kao J, Nikiforovich GV, Achilefu S. Synthesis and characterization of a macrocyclic near-infrared optical scaffold. J Am Chem Soc 2003; 125: 7766-7.

54 Kirtane AR, Kalscheuer SM, Panyam J. Exploiting nanotechnology to overcome tumor drug resistance: Challenges and opportunities. Adv Drug Deliv Rev 2013; 65: 1731-47.

55 Davis ME, Chen ZG, Shin DM. Nanoparticle therapeutics: an emerging treatment modality for cancer. Nat Rev Drug Discov 2008; 7: 771-82.

56 Liu Y, Zhang D, Qiao ZY, Qi GB, Liang XJ, Chen XG, et al. A Peptidenetwork weaved nanoplatform with tumor microenvironment responsiveness and deep tissue penetration capability for cancer therapy. Adv Mater 2015; 27: 5034-42.

57 Wei T, Liu J, Ma H, Cheng Q, Huang Y, Zhao J, et al. Functionalized nanoscale micelles improve drug delivery for cancer therapy in vitro and in vivo. Nano Lett 2013; 13: 2528-34.

58 Hoyer J, Neundorf I. Peptide vectors for the nonviral delivery of nucleic acids. Acc Chem Res 2012; 45: 1048-56.

59 Wagner E. Polymers for SiRNA delivery: inspired by viruses to be targeted, dynamic, and precise. Acc Chem Res 2012; 45: 1005-13.

60 Rizzuti M, Nizzardo M, Zanetta C, Ramirez A, Corti S. Therapeutic applications of the cell-penetrating HIV-1 Tat peptide. Drug Discov Today 2015; 20: 76-85.

61 Liu J, Zhao Y, Guo Q, Wang Z, Wang H, Yang Y, et al. TAT-modified nanosilver for combating multidrug-resistant cancer. Biomaterials 2012; 33: 6155-61.

62 Majzoub RN, Chan CL, Ewert KK, Silva BF, Liang KS, Jacovetty EL, et al. Uptake and transfection efficiency of PEGylated cationic liposomeDNA complexes with and without RGD-tagging. Biomaterials 2014; 35: 4996-5005.

63 Chen X, Wang X, Wang Y, Yang L, Hu J, Xiao W, et al. Improved tumortargeting drug delivery and therapeutic efficacy by cationic liposome modified with truncated bFGF peptide. J Control Release 2010; 145: 17-25.

64 Li Y, Deng Y, Tian X, Ke H, Guo M, Zhu A, et al. Multipronged design of light-triggered nanoparticles to overcome cisplatin resistance for efficient ablation of resistant tumor. ACS Nano 2015; 9: 9626-37.

65 Wang YC, Shim MS, Levinson NS, Sung HW, Xia YN. Stimuliresponsive materials for controlled release of theranostic agents. Adv Funct Mater 2014; 24: 4206-20.

66 Zhuang X, Ma X, Xue X, Jiang Q, Song L, Dai L, et al. A PhotosensitizerLoaded DNA Origami Nanosystem for Photodynamic Therapy. ACS Nano 2016; 10: 3486-95.

67 Wang T, Wang D, Yu H, Wang M, Liu J, Feng B, et al. Intracellularly acid-switchable multifunctional micelles for combinational photo/ chemotherapy of the drug-resistant tumor. ACS Nano 2016; 10 : 3496-508.

68 He C, Liu D, Lin W. Nanomedicine applications of hybrid nanomaterials built from metal-ligand coordination bonds: nanoscale metal-organic frameworks and nanoscale coordination polymers. Chem Rev 2015; 115: 11079-108.

$69 \mathrm{Ng} \mathrm{KK}$, Zheng G. Molecular interactions in organic nanoparticles for phototheranostic applications. Chem Rev 2015; 115: 11012-42.

70 He C, Liu D, Lin W. Self-assembled core-shell nanoparticles for combined chemotherapy and photodynamic therapy of resistant head and neck cancers. ACS Nano 2015; 9: 991-1003. 